\title{
Attentional capture decreases when distractors remain visible during rapid serial visual presentations
}

\author{
TOMOE INUKAI \\ Chukyo University, Nagoya, Japan \\ AND \\ TAKATSUne Kumada AND JUN-ICHIRO KAWAHARA \\ National Institute of Advanced Industrial Science and Technology, Tsukuba, Japan
}

\begin{abstract}
The identification of a central visual target is impaired by the onset of a peripheral distractor. This impairment is said to occur because attentional focus is diverted to the peripheral distractor. We examined whether distractor offset would enhance or reduce attentional capture by manipulating the duration of the distractor. Observers identified a color singleton among a rapid stream of homogeneous nontargets. Peripheral distractors disappeared 43 or $172 \mathrm{msec}$ after onset (the short- and long-duration conditions, respectively). Identification accuracy was greater in the long-duration condition than in the short-duration condition. The same pattern of results was obtained when participants identified a target of a designated color among heterogeneous nontargets when the color of the distractor was the same as that of the target. These findings suggest that attentional capture consists of stimulus onset and offset, both of which are susceptible to top-down attentional set.
\end{abstract}

The visual system must focus attention on a specific region of a complex visual scene when searching for a target, because the system cannot process all information, both relevant and irrelevant, without becoming overwhelmed. Research has shown that the system usually uses one of the two major ways to control attentional focus in visual target selection (Egeth \& Yantis, 1997; Ruz \& Lupiáñez, 2002). The first, endogenous control, refers to instances in which deployment of attentional focus can be controlled intentionally by top-down knowledge. However, attentional focus can also be directed exogenously (bottom-up) to a location in response to an abrupt change.

Attentional capture, the second process underlying attentional control, is characterized by two important aspects, the interaction of which the present study examined. First, attentional capture has been shown to result from many different kinds of transient signals (e.g., abrupt onset, Yantis \& Jonides, 1984; offset, Pratt, Theeuwes, \& Donk, 2007; and luminance change, Franconeri, Hollingworth, \& Simons, 2005). These studies showed that identification of a target among multiple nontargets was enhanced when the target was a uniquely salient item and identification deteriorated when one of the nontarget items was a salient distractor, as compared with cases in which no items were salient (e.g., Theeuwes, 1992). This enhancement/deterioration effect has been explained in terms of the uniquely salient item's capturing the atten- tional focus. When the most salient item happened to be the target, the process of identifying the target could be initiated immediately, because the item was at the center of focused attention. When the most salient item was a distractor, however, attention was unintentionally drawn to the distractor. In this case, correct identification required disengaging focus from the distractor and reallocating it to the target. This disengagement and reallocation of focus requires time (Fischer, 1986), and the original misallocation of attentional focus results in lengthened reaction times and/or increased error rates with regard to target identification.

A second critical aspect of attentional capture is that it can be modulated by top-down knowledge. Research has shown that the occurrence of attentional capture depends on participants' current behavioral goal (e.g., Folk, Leber, \& Egeth, 2002; Folk, Remington, \& Johnston, 1992). For example, Folk et al. (2002) measured identification accuracy for a target (e.g., a red letter) among a rapid sequence of nontargets. A frame of four peripheral distractors (“\#”s) was inserted into the sequence so that one of the distractors was a colored oddball (e.g., a red or green \#). The accuracy with which the target was identified was impaired the most, as compared with cases in which no distractor appeared, when the distractor appeared about $160 \mathrm{msec}$ before the onset of the target and immediately disappeared (the distractor remained on screen for $42 \mathrm{msec}$ ). Critically,

J. Kawahara, jun.kawahara@aist.go.jp 
the pattern of the impairment was contingent on the goal of the observers. When the observers were searching for a red feature embedded in a sequence of nontargets with heterogeneous colors, accuracy of identification was impaired only by the peripheral singleton distractor with the same color as the target (i.e., red). On the other hand, accuracy was impaired by a singleton distractor of any color when the observers were searching for an oddball among homogeneously colored nontargets (e.g., gray). These two different search modes have been called the feature search mode and the singleton detection mode, respectively.

As was noted above, evidence that stimulus transients elicit capture and that two search modes are available has been accumulating. However, the interplay of these aspects has not been investigated systematically. Most studies examining the effect of stimulus transients on attentional capture used spatial visual search tasks and did not address the question of whether the effect of transients was modulated differently under the two types of search modes. Therefore, the present study focused on this potential interaction.

Among the several possible transient stimulus events, the present study focused on stimulus offset, because it is intriguing and because varied predictions can be offered about the disengagement of attentional focus from distractors. On the one hand, stimulus offset could facilitate disengagement of attention. It has been shown that attentional shift from a fixation point to a target is faster when the fixation point disappears than when it remains on screen (gap effect; Mackeben \& Nakayama, 1993). This suggests that the disappearance of the fixation point allows attention to disengage and begin moving to the next location (Godijn \& Kramer, 2007; Loetscher \& Brugger, 2007). This finding was based on the observation that the offset of a fixation point reduced saccade latency to a peripheral target when the point disappeared $200 \mathrm{msec}$ before the target appeared, as compared with cases in which the point remained visible. Because the literature has established that the allocation of attention precedes saccade eye movement, the gap effect has often been explained in terms of attentional disengagement from the fixation point due to its offset (e.g., Fischer \& Weber, 1993; Pratt, Lajonchere, \& Abrams, 2006). In the same vein, inhibition of return has been shown to occur more quickly when the spatial cue disappears than when it remains (Samuel \& Weiner, 2001).

On the other hand, stimulus offset might disturb disengagement because offset itself causes attentional capture (e.g., Atchley, Kramer, \& Hillstrom, 2000; Miller, 1989; Pratt et al., 2007; Watson \& Humphreys, 1995). For example, Atchley et al. asked observers to identify a target letter that was either presented directly or revealed by filler line segments' offsetting to reveal letters. When targets were defined by an offset transient, attention was captured by noninformative precues defined by a stimulus offset. Therefore, when observers were monitoring items in a rapid visual stream, offset transients were able to exaggerate attentional capture. Given the existence of two inconsistent observations regarding stimulus offset and attentional disengagement, and given that the effect of offset on attentional capture has not been tested in the context of contingent attentional capture, the present study contrasted the following two possibilities. First, if the offset of the distractor facilitated disengagement of attention from distractors (Mackeben \& Nakayama, 1993), the degree to which attention is captured would be expected to decrease when the distractor disappeared shortly after its onset versus when it was presented for a longer period. On the other hand, if the offset of the distractor interfered with the disengagement and reengagement of attention (Atchley et al., 2000; Miller, 1989; Pratt et al., 2007; Watson \& Humphreys, 1995), the amount of capture would increase when the distractor disappeared shortly after onset versus when it remained for a longer period of time.

We manipulated attentional sets to specify the kind of information that was searched under these two modes. Specifically, if the amount of attentional capture increased under one of the search modes when the distractor disappeared quickly, this finding would suggest that the offset signal triggered purely bottom-up attentional capture. It is reasonable that, when observers use the singleton detection mode, any salient event, including peripheral transient onset and offset events, will be detected, because this mode will be consistent with the attentional set's relying on relative saliency. However, it is unclear whether the same principle would apply to the feature search mode. If the disappearance of a peripheral distractor did not affect the amount of attentional capture, it would suggest a purely top-down process for the feature search mode that excludes any irrelevant events inconsistent with the current behavioral goal. The finding of an effect on attentional capture would imply that the feature search mode permitted interference by a bottom-up signal. Manipulation of the search modes enabled the study to answer these questions.

\section{EXPERIMENT 1}

This experiment was designed to examine whether the transient change arising from distractor offset would interfere with the accurate identification of the central target when observers adopted two different search modes. To this end, we factorially manipulated the duration of the peripheral distractors and the search modes. The distractors could be presented for a short (43-msec) or relatively long (172-msec) period. This factor was manipulated as a within-subjects variable. The search mode, which was manipulated as a between-subjects variable, could be the singleton detection or the feature search mode. Two predictions can be made with regard to the effect of extending the duration of the distractor. If the offset of the distractor facilitates attentional disengagement, the impairment in target identification due to attentional capture can be expected to decrease when the distractor disappears shortly after its onset versus when it remains visible for a longer period. However, if the offset of the distractor interferes with the disengagement and reengagement of attention, attentional capture will be enhanced when the distractor disappears shortly after onset versus when it remains for a longer period of time. The singleton detection and the feature search modes were induced in Experiments 1A and $1 \mathrm{~B}$, respectively. 
A
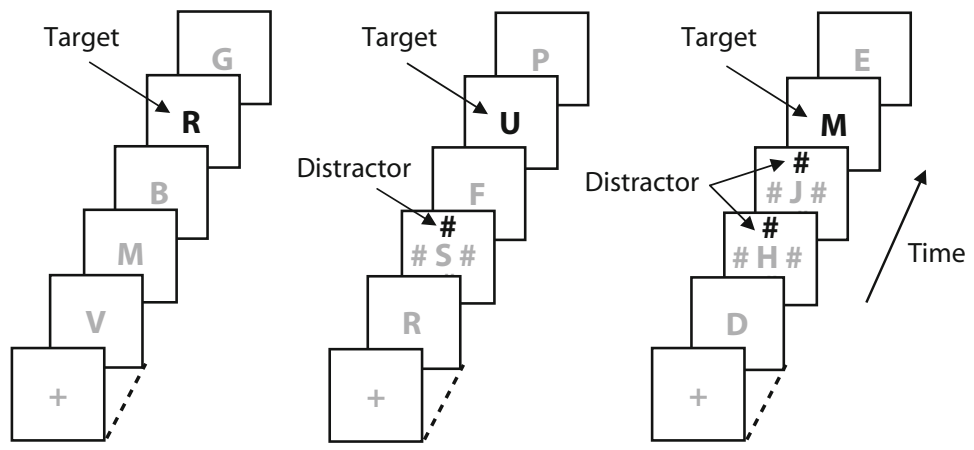

B

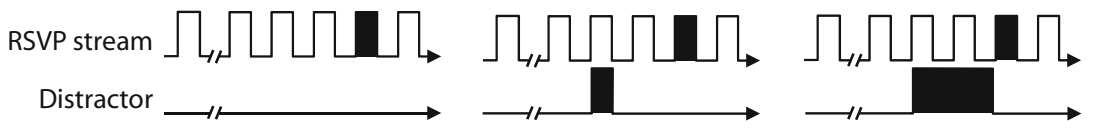

Figure 1. (A and B) Sequences of events in the no-distractor, short-duration, and longduration conditions (from left to right) in Experiment 1A. RSVP, rapid serial visual presentation.

\section{Experiment 1A The Singleton Detection Mode}

\section{Method}

Observers. Sixteen undergraduate students from the National Institute of Advanced Industrial Science and Technology (AIST, Tsukuba, Japan) subject pool participated in exchange for monetary remuneration. All the observers reported normal or corrected-tonormal visual acuity and normal color vision and were naive with respect to the purpose of the experiment.

Apparatus and Stimuli. The stimuli were displayed on a CRT monitor controlled by a PC/AT-compatible computer. The viewing distance was approximately $60 \mathrm{~cm}$. The nontarget sequence consisted of uppercase gray letters randomly chosen in every trial from the English alphabet, without replacement, excluding I, O, Q, and $\mathrm{Z}$. One of five possible letters in the sequence was uniquely colored (red, blue, orange, magenta, and yellow were used for half the observers; green was used instead of red for the other half) and was designated as the target. The letters subtended approximately $1.0^{\circ}$ of visual angle in height and width $\left(\right.$ stroke $\left.=0.1^{\circ}\right)$. The stimulus display consisted of a central fixation cross and a stream of letters in the center of the display (see Figure 1). The stimuli were displayed on a black background. The distractors, when present, consisted of four pound signs (i.e., a \#; $1.0^{\circ}$ in height and width, stroke $=0.1^{\circ}$ ), centered $5.2^{\circ}$ above, below, to the right, and to the left of the fixation cross. Three of the four distractor items were colored gray, and the remaining one was colored either red or green. The target color, the color of the unique distractor, and the location of the unique distractor were randomly determined at the beginning of every trial.

Procedure and Experimental Design. The procedure was essentially identical to that used by Leber and Egeth (2006), except that two distractor durations ( 43 or $172 \mathrm{msec}$ ) were used. Observers initiated each trial by pressing the space bar. After a delay of $500 \mathrm{msec}$, a stream of nontargets was presented. Each trial consisted of 11 to 15 leading items and a target item. Each item in the stream was displayed for $43 \mathrm{msec}$, with an interstimulus interval of $43 \mathrm{msec}$. The number of leading items was determined randomly for all trials. The distractors (the four \#s), when presented, appeared $172 \mathrm{msec}$ before the onset of the target and lasted $43 \mathrm{msec}$ on one third of the trials and $172 \mathrm{msec}$ on another third of the trials. In the remaining one third of the trials, no distractors were included. When the distractors were presented, one of the \#s was either red or green. The target was followed by four to eight nontarget frames. The targets were degraded by superimposing black noise dots $(n=22 ; 1$ pixel each) to prevent accuracy from reaching a ceiling performance. The observers identified the target (the uniquely colored letter in a stream) by typing the corresponding key on the keyboard. We conducted 24 practice trials prior to the 360 experimental trials.

One between- and two within-subjects factors were used. The between-subjects factor was target color (red or green). The withinsubjects factors were distractor duration $(43$ or $172 \mathrm{msec}$ ) and distractor type (same, different, or no). In this context, same referred to cases in which the color of the unique \# was included in the list of the potential target colors. For example, this included conditions in which the unique \# was red and this color was one of the potential target colors. In contrast, a different distractor referred to cases in which the color of the unique \# was not included in the list of the potential target colors. This was the case when the unique \# was green and this color was not included in the list of the potential target colors. The trials without distractor conditions appeared identical, regardless of whether the distractor durations were short or long, because no distractors were involved under these conditions (i.e., the labels of short and long were nominally assigned under the nodistractor condition).

\section{Results}

The results from the red- and green-target groups were collapsed because no main effects or interactions were observed in the analyses, or in all subsequent experiments, regarding target color. Mean percentages of correct responses for each condition are presented in Figure 2A. An ANOVA with distractor type (same, different, or no) and duration (43 or $172 \mathrm{msec}$ ) as within-subjects variables revealed significant main effects for distractor type $[F(2,30)=16.84, p<.001]$ and duration $[F(1,15)=$ $17.14, p<.001]$. The interaction between these factors was also significant $[F(2,30)=6.44, p<.005]$. Multiple comparisons on the main effect of distractor type using Ryan's (1960) method indicated that accuracy in the nodistractor condition was higher than that in the other two conditions $[t \mathrm{~s}(30)>4.86, p \mathrm{~s}<.001]$. We found no significant difference between the same- and different-color 
A

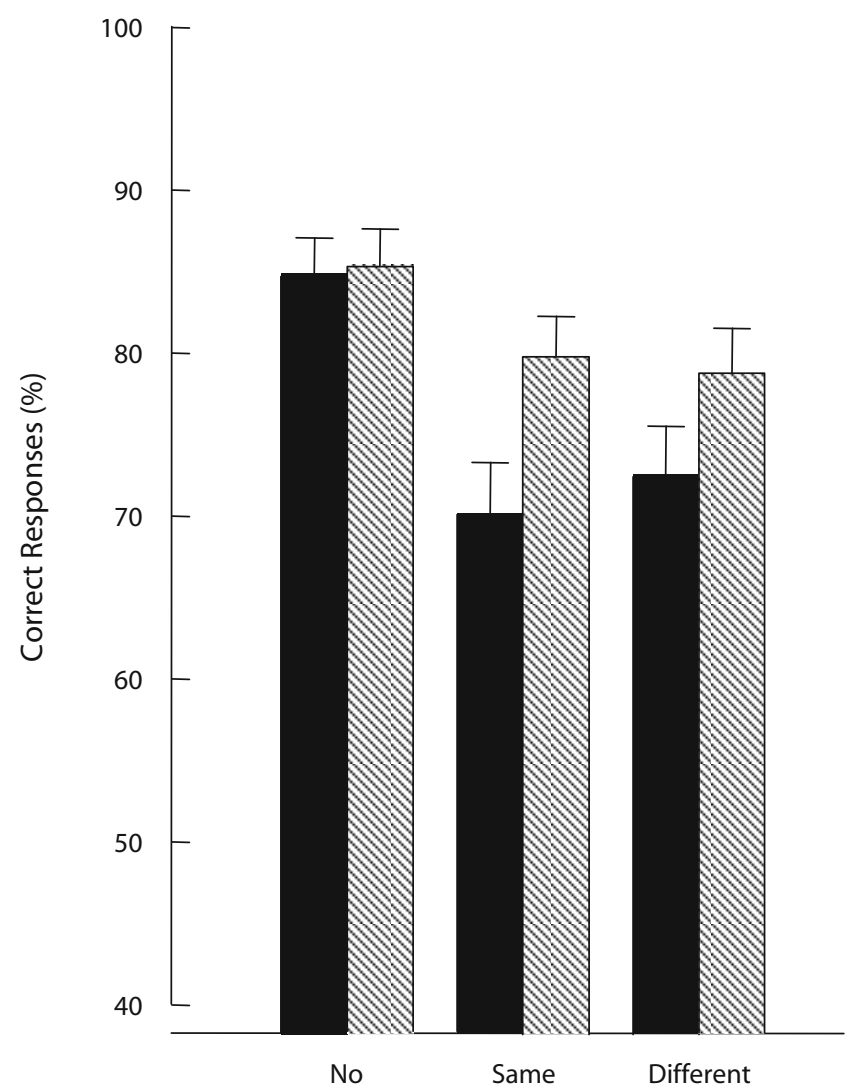

Distractor Condition
B

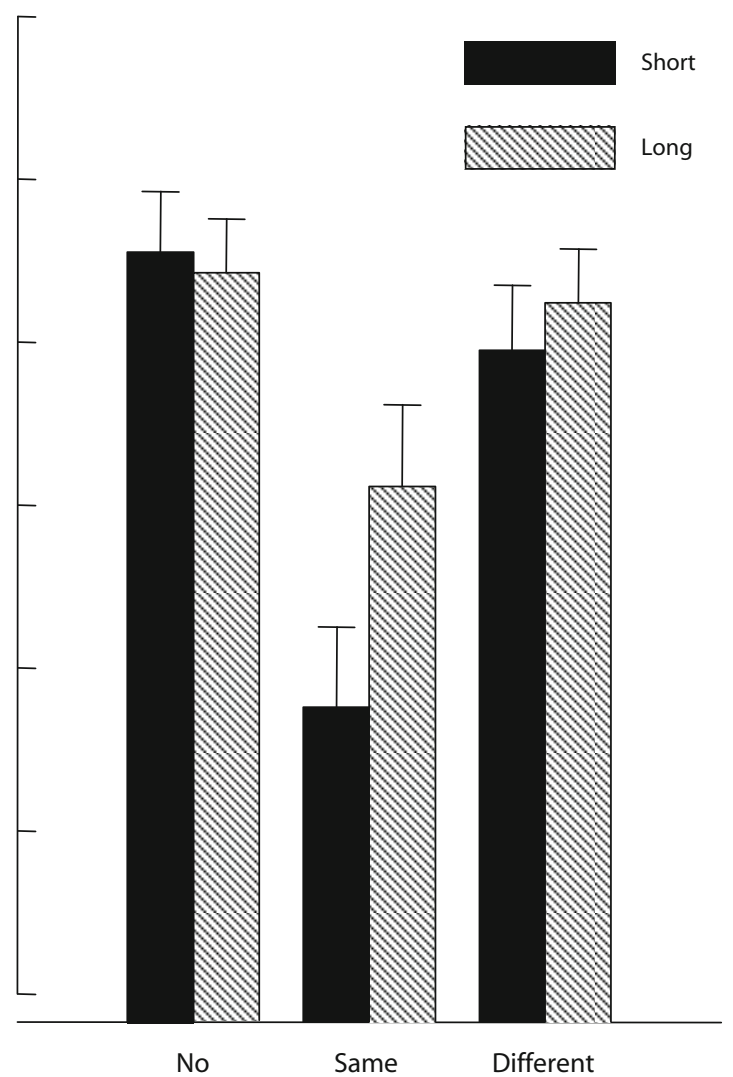

Distractor Condition

Figure 2. (A) Mean percentages of correct target identification in each duration condition (short or long) and for each distractor type (no distractor, same color, or different color) in Experiment 1A. (B) Mean percentages of correct target identification in each duration condition (short or long) and for each distractor type (no distractor, same color, or different color) in Experiment 1B. Error bars indicate standard errors of the means.

distractor conditions $[t(30)=0.32$, n.s.]. Multiple comparisons of the interaction indicated that the accuracy in the same- and different-color conditions was higher when the distractor duration was long than when the duration was short $\left[F_{\mathrm{s}}(1,45)>10.15, p \mathrm{~s}<.005\right]$. However, such differences were not observed in the absence of distractors $[F(1,45)<1.0$, n.s.]. The results presented below collapse distractor types (same or different) because no significant differences were observed with regard to the same- and different-color conditions or in the nodistractor condition.

An ANOVA of accuracy indicated a main effect of distractor condition [no, short, or long distractors; $F(2,30)=$ 23.94, $p<.001]$. Multiple comparisons using Ryan's (1960) method indicated that the accuracy was lowest when the distractor duration was short $[t(30)=6.89, p<$ $.001]$. Note that accuracy was significantly higher when the duration was long than when the duration was short $[t(30)=4.02, p<.001]$. Accuracy was higher in the absence of distractors than it was when the distractor duration was long $[t(30)=2.87, p<.01]$.

\section{Discussion}

In this experiment, we sought to examine whether transient changes due to distractor offset would contribute to the interference effect, impinging on accuracy in identifying the central target. We reasoned that if an ordinary attentional capture effect, as was observed with the present procedure, simply reflected capture by distractor onset, the duration of the peripheral distractors should not affect the results. The results clearly excluded this possibility. Two major findings emerged from the present experiment. First, accuracy rates were lower when distractors were presented than when distractors were not presented, suggesting that we replicated a standard attentional capture effect (Folk et al., 2002; Leber \& Egeth, 2006). Results showing equivalent rates of accurate identification in the same- and different-color conditions suggested that the distractors captured attention regardless of the congruency between the distractor color and the target color. This was consistent with the idea that attention is misallocated to an oddball item when observers are searching for uniqueness (Bacon \& Egeth, 1994). Therefore, the present finding 
indicated that the observers engaged in this task under the singleton detection mode.

Second, identification accuracy was higher when distractor duration was long than when it was short. This set of findings is inconsistent with the idea that the offset promoted the disengagement of attention from the distractor (e.g., Fischer \& Weber, 1993; Godijn \& Kramer, 2007; Mackeben \& Nakayama, 1993; Samuel \& Weiner, 2001). We suggest that this superiority in target identification in the long-duration condition reflects the impact of the ostensibly irrelevant offset of the distractor in the shortduration condition, in that the letter prevented attention from being reallocated to the target location, as if attention were anchored by the offset, even though the observers were able to detect the onset of an oddball color in the central stream. This implies that the offset of a distractor is not subject to top-down attentional control settings. It is reasonable to assume that any salient event, such as the disappearance of a distractor at the periphery, could capture attention under the singleton detection mode insofar as that mode is characterized by sensitivity to salient signals. However, it remained unclear whether the disappearance of a distractor would have a similar effect on attentional disengagement from the distractor under the feature search mode. In Experiment 1B, therefore, we examined whether the offset of a distractor would capture attention when observers searched for the onset of a specific target color.

\section{Experiment 1B}

\section{The Feature Search Mode}

To examine whether the anchoring of attention by distractor offset was subject to top-down attentional control settings, we embedded a target among nontargets of various colors. In this context, observers needed to adopt the feature search mode to detect the onset of a target in a specific color (e.g., a red letter). If the offset of the distractor did not capture attention under the feature search mode, the accuracy with which targets were identified would be comparable under the short- and long-duration distractor conditions. In contrast, if the offset of the distractor captured attention, the accuracy with which the target was identified would be lower under the short-duration condition than it would be under the long-duration condition. If the former were the case, the interference effect would reflect purely top-down capture caused by the onset of the task-relevant feature, whereas if the latter were the case, the interference effect would reflect top-down (i.e., contingent) capture caused by its onset and bottom-up capture caused by its offset.

\section{Method}

The apparatus, stimuli, procedure, and design were the same as those used in Experiment 1A, with the exception of the following changes. The color of the target was consistently red, and the color of each nontarget was chosen from four possible colors (gray, blue, yellow, and green) so that the same color would not appear in successive frames. The colors were equiluminant $\left(21 \mathrm{~cd} / \mathrm{m}^{2}\right.$, as measured by a Minolta CS-100A photometer). Due to the increment in task difficulty, no noise dots were superimposed on the target. One of the peripheral distractors was red or green, in the same- and different-color distractor conditions, respectively. Sixteen undergraduate students from the AIST pool participated in exchange for remuneration. All reported normal or corrected-to-normal visual acuity and normal color vision. They were naive with respect to the purpose of the experiment.

\section{Results}

The mean percentages of correct responses in each condition are presented in Figure 2B. An ANOVA with distractor type (same, different, or no) and duration (43 or $172 \mathrm{msec}$ ) as within-subjects variables revealed significant main effects of distractor type $[F(2,30)=29.1, p<$ $.001]$ and duration $[F(1,15)=17, p<.001]$. The interaction between these factors was also significant $[F(2,30)=$ $16.65, p<.001]$. Multiple comparisons on the main effect of distractor type indicated that accuracy in the same-color condition was lower than that in the other two conditions $[t \mathrm{~s}(30)>5.8, p \mathrm{~s}<.01]$. We found no significant difference between the different-color and no-distractor conditions $[t(30)=1.39$, n.s.]. Multiple comparisons of the interaction indicated that accuracy rates under the samecolor condition were higher when the distractor duration was long than when the duration was short $[F(1,45)=$ $47.73, p<.001]$. However, such differences were not observed in the different-color and no-distractor conditions $[F \mathrm{~s}(1,45)>0.41$, n.s. $]$.

\section{Discussion}

When the observers detected the onset of a specific target color, the accuracy of target identification was impaired only in the same-color condition. Such impairment was not observed in the different-color condition, relative to the no-distractor condition. This result was consistent with that reported by Folk et al. (2002), in that top-down knowledge of the target feature (e.g., red) eliminated attentional capture by a feature of the task-irrelevant distractor (e.g., green). Note that the magnitude of the capture effect in the short-duration condition was greater than that in the long-duration condition. This pattern of results was identical to that found when the observers detected the onset of a target oddball in Experiment 1A. The present results suggest that the focus of the observers' attention on the onset of a specific target color did not eliminate interference by the offset of the distractor. Importantly, we found that attentional deployment to the distractor was contingent on the observers' task set. Impaired target identification was not observed in the different-color condition when the distractor duration was short. Therefore, these results suggest that stimulus-driven signals from the offset of the distractor exert their effect in conjunction with the top-down attentional set; the offset signals interfere with target identification only when the target agrees with the current set.

We suggest that an attentional capture effect was elicited by the peripheral distractors via the spatial diversion of attention caused by the distractors' onset and immediate offset, which occurred at the same location. This scenario involves the distractor offset's anchoring the attentional focus that was about to be disengaged from the distractor and reengaged with the central stream. According to this 
scenario, when the distractor remains on the screen, the attentional focus is easily disengaged and reengaged with the central stream until the target appears.

The results of Experiments 1A and 1B indicated that attentional capture decreased when the duration of the distractors was long, as compared with when it was short. However, Experiments 1A and 1B reflected a confounding between the duration of the distractor and its informativeness with regard to the onset of the target. That is, the long distractor differed from the short distractor in terms of duration, but the offset of the long distractor also coincided with the onset of the target. Therefore, the offset of the distractors might have served as temporal cues for the onset of targets, and this cue might have assisted in target identification only under the long-duration condition. Experiment 2 examined this possibility in the context of searching for an oddball target under the singleton detection mode.

\section{EXPERIMENT 2}

Experiment 2 further manipulated temporal parameters regarding distractor onset and offset. Experiment 2A was designed to test the possibility that the reduced attentional capture observed in the long-duration condition in Experiment 1 occurred because the offset of the long-duration distractor accompanied the onset of the target, since the observers could use the temporal contiguity as a cue for target arrival. To examine this possibility, we removed the temporal contiguity of the distractor offset and the target onset in the long-duration distractor condition by extending the duration, so that the long-duration distractor frame remained on the display until the end of the central stream. Therefore, if the observers used the offset-onset contiguity in the long-duration distractor condition in Experiment 1 as a temporal cue, such a reduction effect in the long-duration distractor condition would not be obtained. In contrast, if the observers did not use the contiguity and, instead, the reduced capture effect reflected an easier attentional dis- engagement from the distractor due to the lack of offset, we should find the same reduced attentional capture as that observed in Experiment 1. The latter would occur because the short-duration distractors included onset and offset transients that captured attention, whereas the long-duration distractors included only onset transients. Thus, fewer transients occurred in the long-duration condition and disengaging attention from the peripheral distractors would have been easier than in the short-duration condition.

\section{Experiment 2A}

\section{Method}

The apparatus, stimuli, and procedures were the same as those in Experiment 1, except that the distractors under the long-duration condition remained in the display until the central rapid serial visual presentation stream ended (on average, $688 \mathrm{msec}$ ). The shortduration distractor condition was identical to that in Experiment $1 \mathrm{~A}$. A schematic diagram of the stimulus display is shown in Figure 3. Sixteen undergraduate students from the AIST subject pool participated in exchange for remuneration. All reported normal or corrected-to-normal visual acuity and normal color vision. They were naive with respect to the purpose of the experiment.

\section{Results}

Mean percentages of correct responses for each condition (no, short-duration, or long-duration distractors) are presented in Figure 4. An ANOVA of the accuracy indicated a main effect of distractor condition $[F(2,30)=$ $19.80, p<.001]$. Multiple comparisons using Ryan's (1960) method indicated that accuracy was significantly higher when the duration was long than when the duration was short $[t(30)=3.57, p<.01]$. Accuracy was higher in the absence of distractors than when the distractor duration was long $[t(30)=2.7, p<.05]$.

\section{Discussion}

The pattern of the results was identical to that obtained in Experiment 1: Attentional capture by the peripheral distractors decreased when the distractor duration was long.

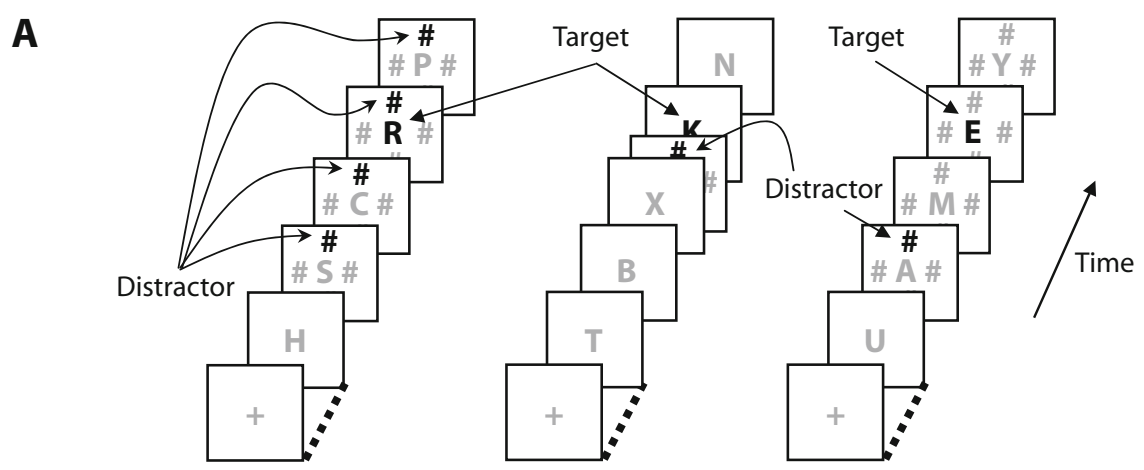

B

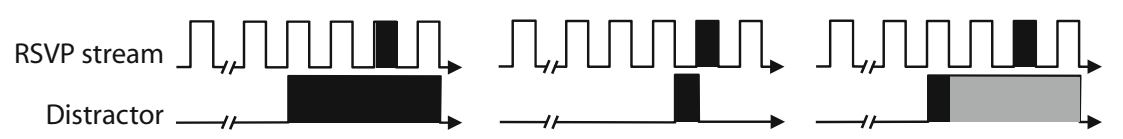

Figure 3. (A and B) Sequences of events in the long-duration, immediately before, and longduration conditions (from left to right) in Experiments 2A, 2B, and 3, respectively. RSVP, rapid serial visual presentation. 


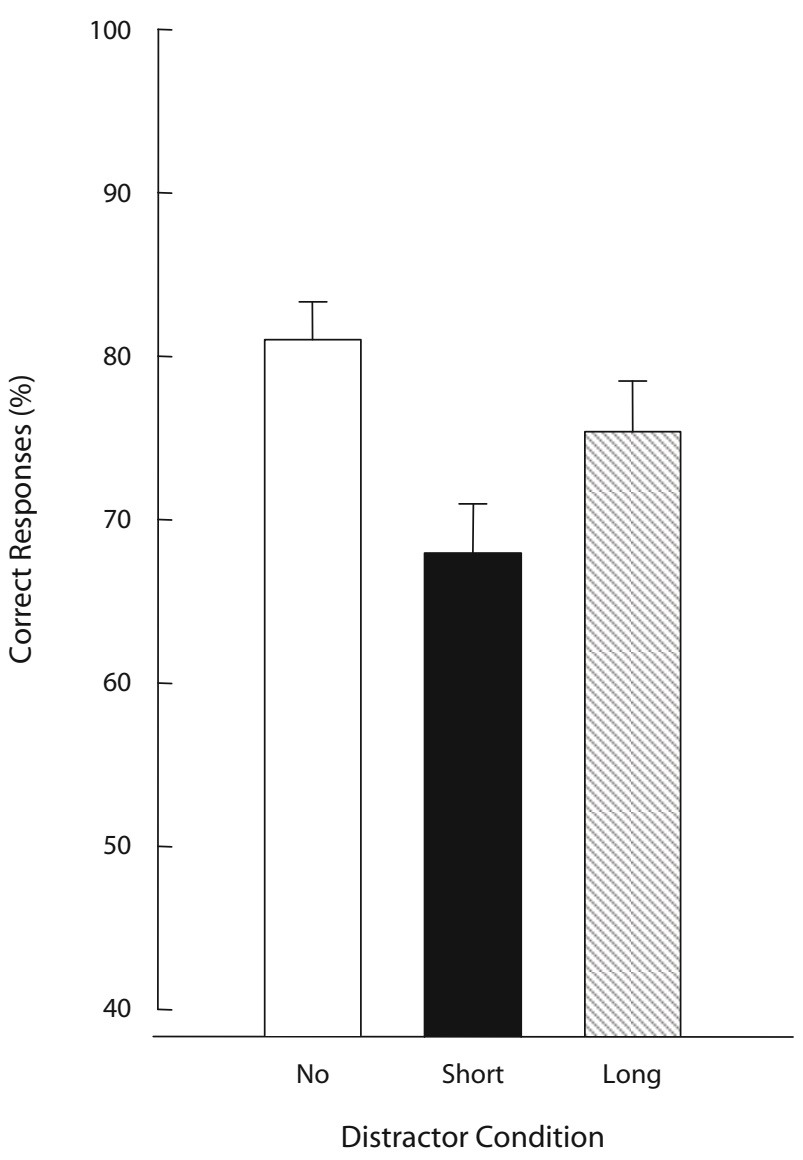

Figure 4. Mean percentages of correct target identification in each distractor condition (no distractor, short duration, or long duration) in Experiment 2A. Error bars indicate standard errors of the means.

This suggests that the coincidence between the offset of distractors and the onset of the target in the long-duration distractor condition in Experiment 1 did not contribute to the reduced capture effect. Rather, the results suggest that the interference effect of peripheral distractors in this paradigm reflected attentional capture not only by the onset of distractors, but also by their offset. This second attentional capture by the distractor offset delayed disengagement of attentional focus from the distractor, resulting in the failure of target identification in the central stream.

In other words, the results of Experiment 2A were consistent with the notion that the interference effect of peripheral distractors included onset and offset transients, because the offset transient in the long-duration condition of Experiment 2A was delayed until the end of the stream; thus, offset did not occur immediately after onset. If the immediate onset-offset hypothesis held, the reduction in attentional capture would be observed to the extent that the onset and offset transients were temporally separated.

Before we tested the immediate onset-offset hypothesis in Experiment 3, we explored whether the effect of a short-duration distractor on the magnitude of attentional capture would change when the distractor offset occurred immediately before the target onset. We posed this ques- tion because it was unclear from Experiment 2A whether the effect of short-duration distractors would benefit from an offset that occurred nearer the target, although when the distractor remained unchanged, a smaller capture effect for the long-duration distractors still persisted in the experiment. Therefore, we pursued this question in Experiment 2B.

\section{Experiment 2B}

We manipulated the temporal proximity of the shortduration distractor to the target in order to examine whether the effect of a short-duration distractor on the magnitude of attentional capture would change when the distractor offset occurred immediately before target onset. This manipulation would be useful for showing the effect of the short-duration distractor on attentional capture when it was presented later in the stream, so that offset occurred just before the target. ${ }^{1}$ If the effect of a short-duration distractor remained the same as that obtained in the preceding experiments when the distractor disappeared just before the target, it would indicate that attentional capture occurs regardless of the distractor-target temporal proximity. In contrast, if the effect of the short-duration distractor was diminished when the distractor disappeared immediately before the target, it would mean that attentional capture decreases when the distractor disappears close to the target. However, even if the latter were the case, such a result might be caused by the temporal proximity of the distractor onset to the target, rather than that of the distractor offset to the target. That is, because the onset of the distractor is too close to the target, the visual system might use attention to process the target before the distractor captures attention. Nonetheless, it was worth examining whether the effect of the short-duration distractor would still be obtained when the offset distractor occurred immediately before the target.

\section{Method}

The apparatus, stimuli, and procedures were the same as those used in Experiment 1A, except for the following changes. Three distractor conditions were used. Under the immediately before condition, the short-duration distractor appeared in the blank interval $(\mathrm{SOA}=43 \mathrm{msec})$ immediately before target onset, so that the offset of the distractor occurred just before target onset (Figure 3). The short-duration and no-distractor conditions were identical to those used in Experiment 1A. Twenty undergraduate students from the AIST subject pool participated in exchange for remuneration. All reported normal or corrected-to-normal visual acuity and normal color vision. They were naive as to the experimental purpose.

\section{Results}

The mean percentages of correct responses for each (no-distractor, short-duration, or immediately before) conditions are presented in Figure 5. An ANOVA of accuracy indicated a main effect of distractor condition $[F(2,38)=8.36, p<.001]$. Multiple comparisons using Ryan's (1960) method indicated that accuracy was lowest $[t \mathrm{~s}(38)>2.71, p \mathrm{~s}<.05]$ in the short-duration condition. The difference in accuracy for the no-distractor and immediately before conditions was not significant $[t(38)=$ $1.3, \mathrm{n} . \mathrm{s}]$. 


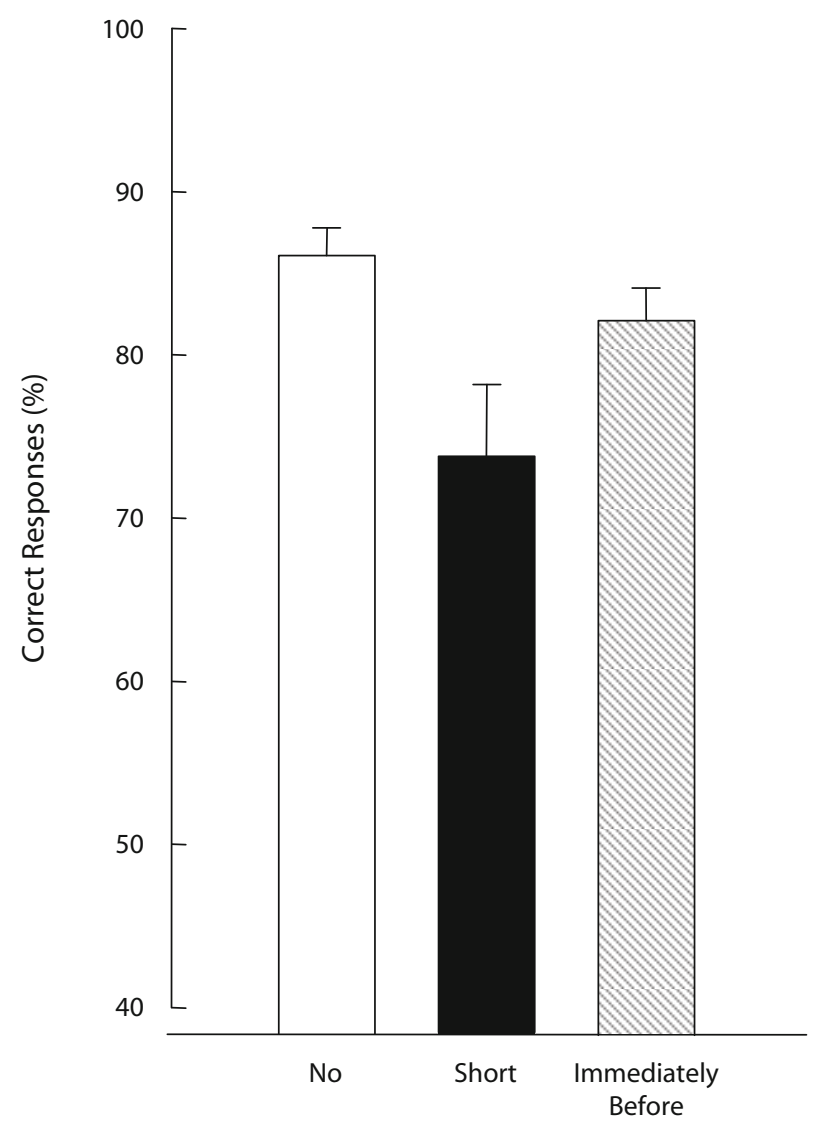

Distractor Condition

Figure 5. Mean percentages of correct target identification in each distractor condition (no distractor, short duration, or immediately before) in Experiment 2B. Error bars indicate standard errors of the means.

\section{Discussion}

When the offset of the short-duration distractor occurred immediately before the target appeared, the distractor did not capture attention. This result suggested that the magnitude of attentional capture varies depending on the temporal proximity between the onset of the distractor and the target onset and is consistent with previous findings (Folk et al., 2002). However, it was unclear whether such a reduction in attentional capture occurred because the distractor disappeared immediately before the target or because the onset of the distractor was temporally too close to the target. In other words, separate manipulations of the duration of the distractor and its temporal proximity to the target caused an inherent problem in that shorter distractor-target lags produced a weaker or even a null capture effect. Therefore, these manipulations were not included in the following experiments.

\section{EXPERIMENT 3}

Experiment 3 tested whether the removal of the offset transient (i.e., disappearance) shortly after the onset of the distractor would reduce attentional capture. In the long- duration condition (Figure 3), the distractor appeared for $43 \mathrm{msec}$ as a red or green singleton and then turned into a gray \# until the end of the stream. Because the three colors (i.e., red, green, and gray) were equiluminant, the impact of the offset transient in the long-duration condition would be greatly reduced, as compared with that in the short-duration condition. If the immediate onset-offset hypothesis were true, the same reduction effect for attentional capture as that observed in Experiments 1 and 2 would be observed.

\section{Method}

The apparatus, stimuli, and procedures were the same as those used in Experiment 2, except that the peripheral colored oddball (a red or green \#) under the long-duration condition remained on the screen for $43 \mathrm{msec}$ and then turned to gray (Figure 3). The three colors - red, green, and gray - were equiluminant $\left(21 \mathrm{~cd} / \mathrm{m}^{2}\right.$, as measured by a Minolta CS-100A photometer). Sixteen undergraduate students from the AIST subject pool participated in exchange for remuneration. All reported normal or corrected-to-normal visual acuity and normal color vision. They were naive with respect to the purpose of the experiment.

\section{Results and Discussion}

Mean percentages of correct responses in each condition (no, short-duration, or long-duration distractors) are

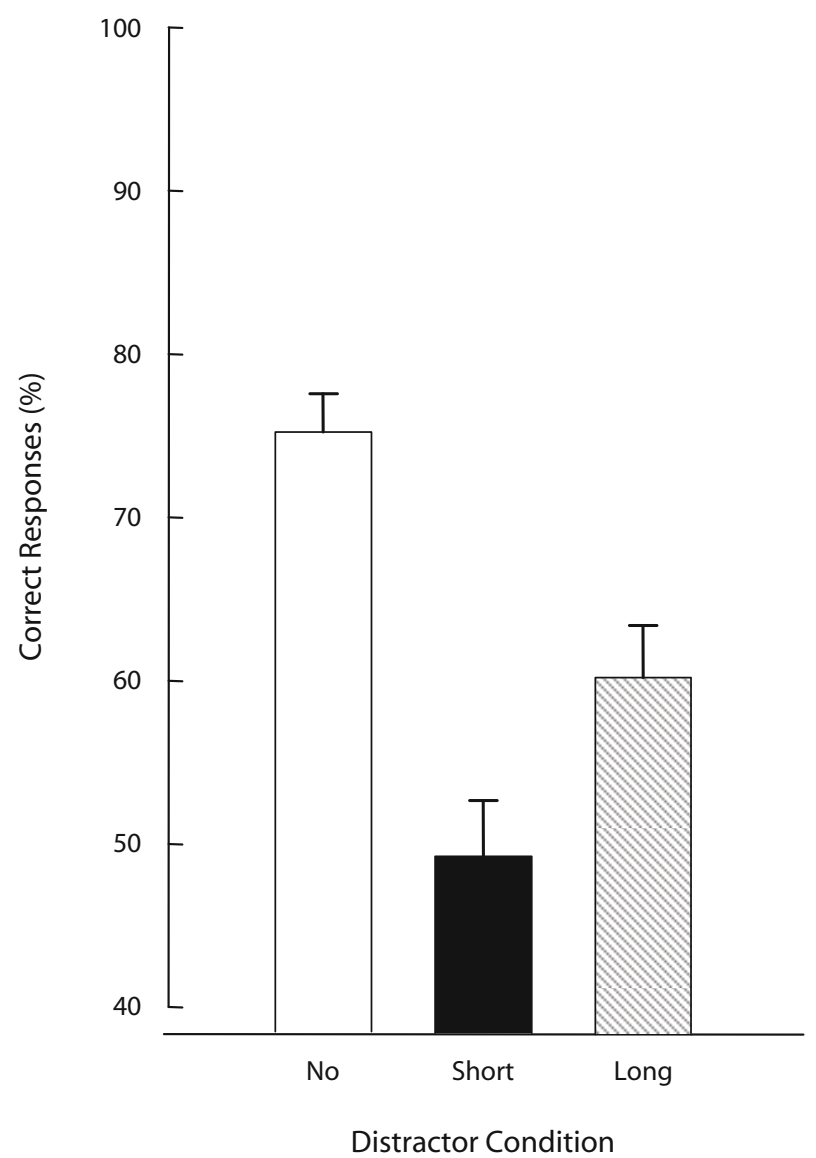

Figure 6. Mean percentages of correct target identification in each distractor condition (no distractor, short duration, or long duration) in Experiment 3. Error bars indicate standard errors of the means. 
presented in Figure 6. An ANOVA of accuracy indicated a main effect of distractor condition $[F(2,30)=46.45, p<$ $.001]$. Multiple comparisons indicated that accuracy in the long-duration condition was significantly higher than that in the short-duration condition $[t(30)=4.04, p<.01]$. Accuracy in the no-distractor condition was higher than that in the long-duration condition $[t(30)=5.56, p<.01]$.

The results were virtually identical to those in Experiments 1 and 2. Attentional capture by the peripheral distractors decreased when the offset transient was reduced by changing the distractor color into equiluminant gray, and the item remained on the screen until the end of the stream. These convergent results were consistent with the idea that the interference effect from the peripheral distractors reflected attentional capture arising from the onset transient of the distractor and immediately (43 msec later) following offset transient.

\section{EXPERIMENT 4}

The results thus far have been consistent with the view that attentional capture, measured as an interference effect from briefly presented peripheral distractors, did not exclusively represent capture derived by the onset of distractors. Rather, this phenomenon represented an amalgam of the attentional capture due to both the onset and the offset transients. Confirmation of this conclusion, however, required resolution of one other issue. That is, the advantage observed in relation to the long-duration distractor might not derive from the temporal contiguity of the onset and offset transients of the distractors but, rather, from the attentional set of the observers. In particular, it has been established that observers adaptively choose an attentional set to be used to detect specific target properties on the basis of task requirements (Folk et al., 2002; Folk et al., 1992; Most \& Astur, 2007) and that distractors matching the set can be selected erroneously. Thus, observers might configure their attentional set to detect a salient colored item with the same duration as that of the central stream (43 msec). If this were the case, the accuracy of target identification would be impaired in the short-duration condition, because the attentional set appropriate for the distractors would match that appropriate for the target insofar as they shared both color and duration; thus, the distractors would be selected erroneously. The distractors in the long-duration condition, however, would not severely impair accuracy, because the attentional set associated with the long-duration distractors would not match that associated with the target.

In Experiment 4, we tested this possibility by introducing a new target duration: $14 \mathrm{msec}$. We hypothesized that if the reduced attentional capture obtained in the previous experiments arose from the use of an attentional set appropriate for detecting a color singleton with a presentation duration identical to that of the target, the longduration distractors would be easier to exclude than the short-duration distractors. In the present experiment, both types of distractors were expected to be effectively excluded equally, because both types (short, $43 \mathrm{msec}$; long,
$172 \mathrm{msec}$ ) did not match the attentional sets appropriate for the target. This hypothesis predicted that the advantage for the long duration would not be obtained when the target duration was $14 \mathrm{msec}$. Alternatively, if the reduced capture were due to the temporal proximity of the onset and offset transients, only the short distractors should interfere with accurate target identification.

\section{Method}

The apparatus, stimuli, and procedure were the same as those used in Experiment 1A, with the exception that a target duration of 14 msec was used. ${ }^{2}$ Sixteen undergraduate students from the AIST subject pool participated in exchange for remuneration. All reported normal or corrected-to-normal visual acuity and normal color vision.

\section{Results and Discussion}

Mean percentages of correct responses for each target duration and distractor condition are presented in Figure 7. An ANOVA with distractor condition (no, shortduration, or long-duration distractors) as within-subjects variables revealed a significant main effect of distractor condition $[F(2,30)=16.42, p<.001]$. Multiple comparisons indicated that accuracy was highest in the no-

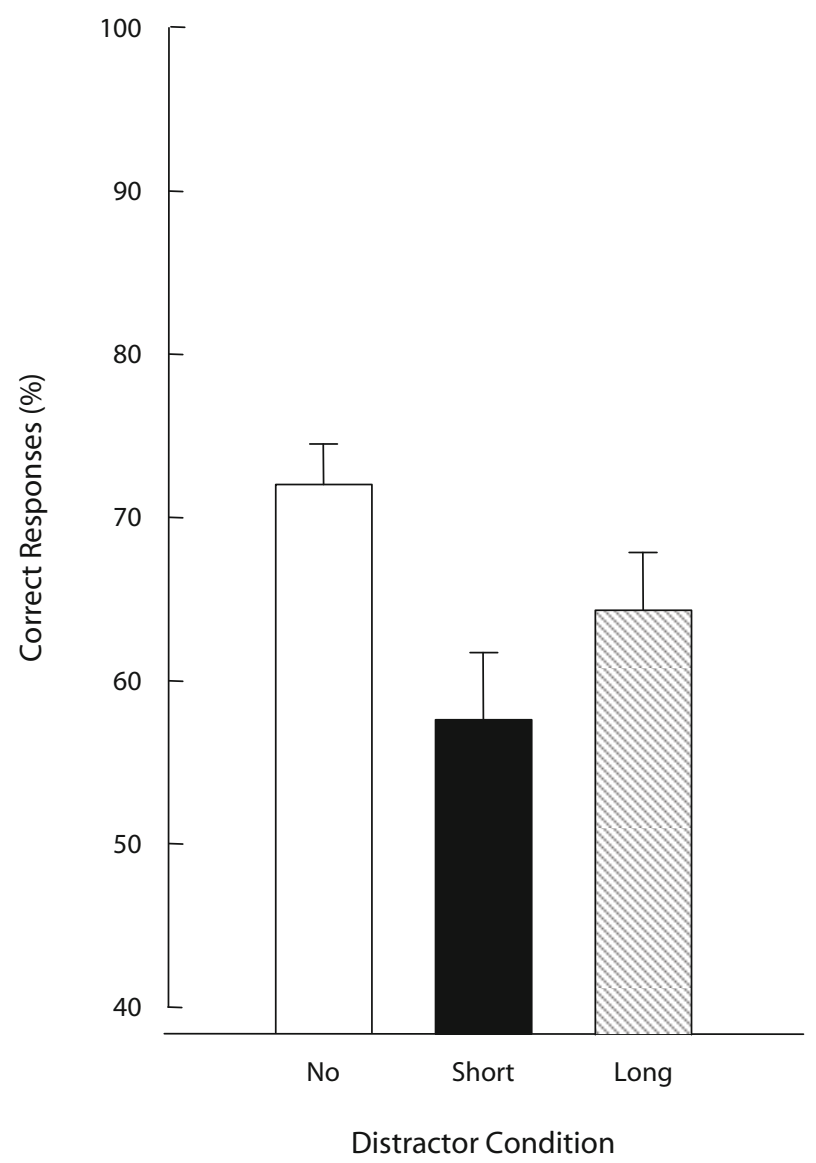

Figure 7. Mean percentages of correct target identification in each distractor condition (no distractor, short duration, or long duration) in Experiment 4. Error bars indicate standard errors of the means. 
distractor condition and lowest in the short-distractor condition $[t \mathrm{~s}(30)>3.06, p \mathrm{~s}<.05]$. Accuracy was significantly lower in the short-duration distractor condition than in the long-duration distractor condition $[t(30)=$ $2.67, p<.05]$.

When target duration was different from both types of distractor duration, the results were identical to those in the previous experiments. That is, accuracy in the short-duration distractor condition was lowest, that in the no-distractor condition was highest, and that in the long-duration distractor condition was intermediate. This pattern of results excluded the possibility that the attentional set for monitoring the specific target duration (43 $\mathrm{msec})$ contributed to the reduced attentional capture in the long-duration condition, relative to that in the shortduration condition. Rather, the present results were consistent with the hypothesis that the greater interference in the short-duration distractor condition, relative to the long-duration distractor condition, arose from the attentional capture due to contiguous transient events (i.e., by the onset and offset of the distractors).

\section{GENERAL DISCUSSION}

The present study involved manipulating the duration of distractors (short, $43 \mathrm{msec}$; long, $172 \mathrm{msec}$ ) to examine whether interference by peripheral distractors on identification of a central target, measured according to the procedure used by Folk et al. (2002), was an amalgam of attentional capture by the onset and offset distractors. We predicted that interference would be affected by distractor duration if disengaging and reengaging processes were involved in this type of attentional capture.

The results clearly indicated that distractor duration affected attentional capture. Note that in all four experiments, attentional capture was consistently smaller when the distractor duration was long than when it was short. This pattern of results contradicts findings indicating that the offset of a distractor facilitates disengaging attention from an item (e.g., Godijn \& Kramer, 2007; Mackeben \& Nakayama, 1993; Samuel \& Weiner, 2001). According to this perspective, the visual system would have quickly reallocated attention to the central stream, because the offset of the distractor would have reduced the time required for disengaging attention from the distractor location. However, this notion did not explain the present finding that attentional capture decreased in the long-duration condition, in which the distractor remained visible.

The reduced attentional capture obtained in Experiment 1 was not an artifact of the temporal coincidence regarding the offset of the long-duration distractor and the onset of the target, and this synchrony might have served as a cue for target identification. Indeed, a similar reduction in attentional capture was observed even when the duration of the long-duration distractor was extended until the end of the central stream, so that the offset of the longduration distractor would not provide any temporal cue (Experiment 2A). In Experiment 3, we found a smaller attentional capture when the transient change due to the distractor offset was moderated. Finally, in Experiment 4, we tested and rejected the alternative hypothesis that the reduced attentional capture was due to the attentional set's being calibrated to search for target items with short durations.

One might argue that the observers selectively inhibited the distractor location in the long-duration condition, whereas they could not use this strategy in the shortduration condition. In the present circumstance, the differences between the two distractor conditions (short vs. long) emerged $43 \mathrm{msec}$ later than distractor onset. It was only $129 \mathrm{msec}$ until the long-duration distractor disappeared from this critical moment, and we believe that endogenous suppression would not be effective during such a short period. As can be seen in the literature, it would take over $300 \mathrm{msec}$ before an endogenous system was activated (e.g., Müller \& Rabbitt, 1989).

We suggest that the reduced attentional capture in the long-duration condition was due to the absence of offset of the peripheral distractor. In other words, attentional capture, measured by the procedure used by Folk et al. (2002), consisted of a mixture of the costs arising from disengaging and reengaging attentional focus, and the offset transient played a critical role in such processes. Given that the observers were spatially focused on the central stream and an onset oddball distractor diverted the focus from the center (Yantis \& Jonides, 1984), attentional focus had to be disengaged from the distractor location and reengaged with the central stream. What follows represents our interpretation of the temporal dynamics characterizing the attentional focus.

When the visual system monitored the distractors preceding the target, attentional focus remained at the center. Following the onset of the distractor frame, focus was diverted to a peripheral singleton location (Yantis \& Jonides, 1984). This occurred because the observers adopted the singleton detection mode (Bacon \& Egeth, 1994) in that context. Although the observers were set to find a color singleton, we believe it is highly likely that the salient items in the temporal domain were able to capture attention. Indeed, when the observers adopted the singleton detection mode, salient items within a different stimulus feature could capture attention in the temporal domain (interdimensional capture; Dalton \& Lavie, 2006; Inukai, Kawahara, \& Kumada, 2010). Since the peripheral items could not serve as targets, the system disengaged the focus from the location of the singleton distractor and started to reengage with the center. Before this disengagement/ reengagement could occur, however, the peripheral distractors in the short-duration condition again captured the focus, for the peripheral location occupied by this offset, which otherwise would soon have been reengaged with the center of the screen (Atchley et al., 2000). ${ }^{3}$ A second capture of the focus did not occur in the long-duration condition, because the offset was scheduled to occur much later (172 msec after the onset) in Experiments 1 and 4, and not to occur until the end of the stream presentation in Experiment 2 . Therefore, the disengagement/reengagement processes were less affected by the distractor offset in the 
long-duration distractor condition, resulting in smaller degrees of attentional capture.

Critically, the present study revealed that the bottomup factor arose from a transient change, such as the offset of a distractor, and affected the top-down attentional capture, contingent on the attentional set of the observers. In the present study, the observers' optimal attentional set involved detection of the onset of a singleton item (Experiments 1A, 2-4; the singleton detection mode) or the onset of an item with a designated target color (Experiment 1B; the feature search mode). Therefore, monitoring the offset of any items was unnecessary. However, distractor offset affected identification accuracy, irrespective of the search mode. Importantly, we found that deploying attention to the distractor was contingent on the observer's task set. Impaired target identification was not observed in the different-color condition when the distractor duration was short. The offset of the distractor, a stimulus-driven signal, did not cause an imperative effect, but it was revealed that the impact of the stimulus offset is contingent on the top-down attentional set: The offset signal interferes with target identification only when the target agrees with the current set. Therefore, we suggest that the attentional capture obtained under the present circumstances was affected by two components: top-down knowledge of specified onset and bottom-up signals that arose from an irrelevant offset. In other words, if no contribution were made by the bottom-up component, the accuracy would not have been affected by distractor duration when top-down attentional capture occurred. Taken together with the finding that stimulus-driven saliency affects attentional capture under the feature search mode (Lamy, Leber, \& Egeth, 2004), the present results suggest that the feature search mode is not driven exclusively by top-down control but is simultaneously affected by bottom-up signals.

A similar effect of distractor duration was observed in the study conducted by Du and Abrams (2008), who showed that accuracy in identifying a target was greater when the distractor was followed by a gray \# (the onsetonly condition) than when the distractor was not followed by a gray \# (the onset-and-offset condition). On the basis of this result, the authors suggested that the effect of the offset of the distractor on attentional capture was not contingent on the task set of observers. However, the reduced capture was confounded with the effect of visual masking. In their study, the saliency of the distractor was reduced by the subsequent gray \# that caused backward masking of the distractor. Although they tried to eliminate this possibility by displacing the location of the gray \# across frames, this still could cause object substitution masking (Lleras \& Moore, 2003; Tata \& Giaschi, 2004). In this sense, the present study clearly demonstrated the effect of the offset of distractors in the absence of confounding related to visual masking.

In summary, the present study highlighted the effect of stimulus offset on attentional capture. We showed that attentional capture decreased when salient distractors remained in the display, relative to when the distractors disappeared shortly after onset. These results suggested that when attentional focus was diverted to the periphery by distractor onset, such focus was disrupted in its disengagement process and was recaptured by the same distractor when offset followed shortly after onset. Such attentional capture by onset and offset transient changes occurred only when the distractor color was consistent with a current search mode, suggesting that attentional capture in the present context was elicited by stimulus onset and offset and that both of these were susceptible to the top-down attentional set.

\section{AUTHOR NOTE}

Correspondence concerning this article should be addressed to T. Inukai, c/o J. Kawahara, National Institute of Advanced Industrial Science and Technology, Central 6, 1-1-1 Higashi, Tsukuba 305-8566, Japan (e-mail: jun.kawahara@aist.go.jp).

\section{REFERENCES}

Atchley, P., Kramer, A. F., \& Hillstrom, A. P. (2000). Contingent capture for onsets and offsets: Attentional set for perceptual transients. Journal of Experimental Psychology: Human Perception \& Performance, 26, 594-606.

BAcon, W. F., \& Egeth, H. E. (1994). Overriding stimulus-driven attentional capture. Perception \& Psychophysics, 55, 485-496.

Dalton, P., \& Lavie, N. (2006). Temporal attentional capture: Effects of irrelevant singletons on rapid serial visual search. Psychonomic Bulletin \& Review, 13, 881-885.

Du, F., \& Abrams, R. A. (2008). Synergy of stimulus-driven salience and goal-directed prioritization: Evidence from the spatial blink. Perception \& Psychophysics, 70, 1489-1503.

Egeth, H. E., \& YanTIS, S. (1997). Visual attention: Control, representation, and time course. Annual Review of Psychology, 48, 269-297.

FISCHER, B. (1986). The role of attention in the preparation of visually guided eye movements in monkey and man. Psychological Research, 48, 251-257.

FISCHER, B., \& WeBer, H. (1993). Express saccades and visual attention. Behavioral \& Brain Sciences, 16, 553-610.

Folk, C. L., Leber, A. B., \& Egeth, H. E. (2002). Made you blink! Contingent attentional capture produces a spatial blink. Perception $\&$ Psychophysics, 64, 741-753.

Folk, C. L., Remington, R. W., \& Johnston, J. C. (1992). Involuntary covert orienting is contingent on attentional control settings. Journal of Experimental Psychology: Human Perception \& Performance, 18, 1030-1044.

Franconeri, S. L., Hollingworth, A., \& Simons, D. J. (2005). Do new objects capture attention? Psychological Science, 16, 275-281.

Godiun, R., \& Kramer, A. F. (2007). Antisaccade costs with static and dynamic targets. Perception \& Psychophysics, 69, 802-815.

Inukai, T., Kawahara, J., \& Kumada, T. (2010). Nonspatial interdimensional attentional capture. Attention, Perception, \& Psychophysics, 72, 658-666.

Lamy, D., Leber, A., \& Egeth, H. E. (2004). Effects of task relevance and stimulus-driven salience in feature search mode. Journal of Experimental Psychology: Human Perception \& Performance, 30, 10191031.

Leber, A. B., \& Egeth, H. E. (2006). Attention on autopilot: Past experience and attentional set. Visual Cognition, 14, 565-583.

Lleras, A., \& Moore, C. M. (2003). When the target becomes the mask: Using apparent motion to isolate the object-level component of object substitution masking. Journal of Experimental Psychology: Human Perception \& Performance, 29, 106-120.

Loetscher, T., \& Brugger, P. (2007). A disengagement deficit in representational space. Neuropsychologia, 45, 1299-1304.

Mackeben, M., \& NaKayAma, K. (1993). Express attentional shifts. Vision Research, 33, 85-90.

Miller, J. (1989). The control of attention by abrupt visual onsets and offsets. Perception \& Psychophysics, 45, 567-571. 
Most, S. B., \& Astur, R. S. (2007). Feature-based attentional set as a cause of traffic accidents. Visual Cognition, 15, 125-132.

MülleR, H. J., \& RabBiTT, P. M. (1989). Reflexive and voluntary orienting of visual attention: Time course of activation and resistance to interruption. Journal of Experimental Psychology: Human Perception \& Performance, 15, 315-330.

Pratt, J., Lajonchere, C. M., \& Abrams, R. A. (2006). Attentional modulation of the gap effect. Vision Research, 46, 2602-2607.

Pratt, J., Theeuwes, J., \& Donk, W. (2007). Offsets and prioritizing the selection of new elements in search displays: More evidence for attentional capture in the preview effect. Visual Cognition, 15, 133-148.

RuZ, M., \& LupiáñEZ, J. (2002). A review of attentional capture: On its automaticity and sensitivity to endogenous control. Psicologica, 23, 283-309.

RYAN, T. A. (1960). Significance tests for multiple comparison of proportions, variances, and other statistics. Psychological Bulletin, 57, 318-328.

Samuel, A. G., \& Weiner, S. K. (2001). Attentional consequences of object appearance and disappearance. Journal of Experimental Psychology: Human Perception \& Performance, 27, 1433-1451.

TATA, M. S., \& Giaschi, D. E. (2004). Warning: Attending to a mask may be hazardous to your perception. Psychonomic Bulletin \& Review, 11, 262-268.

Theeuwes, J. (1992). Perceptual selectivity for color and form. Perception \& Psychophysics, 51, 599-606.

Watson, D. G., \& Humphreys, G. W. (1995). Attention capture by contour onsets and offsets: No special role for onsets. Perception \& Psychophysics, 57, 583-597.

Yantis, S., \& Jonides, J. (1984). Abrupt visual onsets and selective attention: Evidence from visual search. Journal of Experimental Psychology: Human Perception \& Performance, 10, 601-621.

\section{NOTES}

1. We thank Caleb Owen for pointing out the issue.

2. It should be noted that the observers were sensitive enough to distinguish the two short-duration conditions (14 vs. $43 \mathrm{msec})$. In a pilot experiment, observers discriminated the target durations by pressing one of the two designated keys. The mean percentage of correct responses was $70 \%$. A binomial test revealed that accuracy in the two sessions was significantly higher than chance $(p<.001)$, indicating that the observers were able to distinguish these two target durations.

3. It would be interesting to examine what happens when a second capture occurs. One possibility is that the visual system may engage in an additional process to update the current object representations, resulting in poor critical performance. This idea, suggested by an anonymous reviewer, is built upon the findings of Samuel and Weiner (2001), who claimed that rapid disengagement from offsets (relative to onsets) is possible because, after an object offsets, nothing is left to process. In the present study, the color singleton captured attention. In the long-duration condition, attention is captured, the item is processed, and then attention disengages. However, in the short-duration condition, attention is captured, the item is processed, and then the status of the object has to be updated when it disappears. The processing of this additional disappearance event may be what keeps attention from quickly disengaging from the irrelevant distractor, resulting in poor performance for short-duration distractors. Unfortunately, the present experiment was not designed to examine whether the distractor representation was updated upon capture but, rather, to probe whether the distractor interfered with central target identification. Therefore, this intriguing possibility should be tested in future studies.

(Manuscript received August 17, 2008; revision accepted for publication January 10, 2010.) 